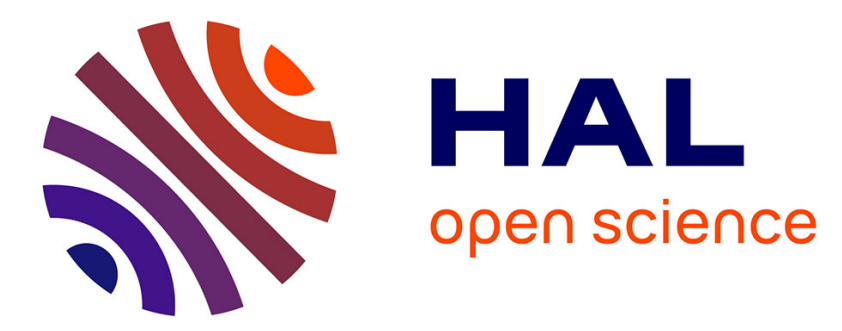

\title{
Coupled non-equilibrium fluctuations in a polymeric ternary mixture
}

L. García-Fernández, P. Fruton, Henri Bataller, J. M Ortiz de Zárate, Fabrizio

Croccolo

\section{- To cite this version:}

L. García-Fernández, P. Fruton, Henri Bataller, J. M Ortiz de Zárate, Fabrizio Croccolo. Coupled nonequilibrium fluctuations in a polymeric ternary mixture. European Physical Journal E: Soft matter and biological physics, 2019, 42 (11), 10.1140/epje/i2019-11889-4 . hal-02473051

\section{HAL Id: hal-02473051 \\ https://hal.science/hal-02473051}

Submitted on 12 Feb 2020

HAL is a multi-disciplinary open access archive for the deposit and dissemination of scientific research documents, whether they are published or not. The documents may come from teaching and research institutions in France or abroad, or from public or private research centers.
L'archive ouverte pluridisciplinaire HAL, est destinée au dépôt et à la diffusion de documents scientifiques de niveau recherche, publiés ou non, émanant des établissements d'enseignement et de recherche français ou étrangers, des laboratoires publics ou privés. 
Coupled non-equilibrium fluctuations in a polymeric ternary mixture L. García-Fernández ${ }^{1,2}$, P. Fruton ${ }^{1}$, H. Bataller ${ }^{1}$, J.M. Ortiz de Zárate ${ }^{3}$ and F. Croccolo ${ }^{1}$

${ }^{1}$ Laboratoire des Fluides Complexes et leurs Réservoirs - IPRA, UMR5150, E2S-Univ Pau \& Pays Adour/CNRS/Total, 1 Allée du Parc Montaury,64600, Anglet, France.

${ }^{2}$ Centre National d'Études Spatiales (CNES),2, Place Maurice Quentin, 75001 Paris, France. ${ }^{3}$ Departamento de Estructura de la Materia, Física Térmica y Electrónica, Facultad de Ciencias Físicas, Universidad Complutense de Madrid. Plaza de las Ciencias 1, 28040 Madrid, Spain.

\section{Abstract}

We investigate by dynamic shadowgraphy the non-equilibrium fluctuations at the steady state of a thermodiffusion experiment in a polymeric ternary mixture of polystyrene-toluene-nhexane. The structure function of the refractive index reveals the existence of quite different decay times, thus requiring the analysis of a wide range of correlation times. This is related to the simultaneous presence of three distinct decay modes corresponding to (from fastest to slowest) relaxation of temperature fluctuations, of the concentration fluctuations of the mixed solvent, and of the concentration fluctuations of the polymer in the binary solvent. An investigation of the decay times at the corresponding diffusive regimes provides a measurement of the thermal diffusivity and the two eigenvalues of the mass diffusion matrix of the ternary mixture. Similar experiments were performed in the past but here, to suppress the confinement effect and obtain a more direct comparison with the theory, a thicker sample is studied. Moreover, also a faster camera is used allowing the experimental observation of faster modes, like the propagative ones. The experimental values of the decay times are eventually compared with those predicted by different available theories. Finally, we present a more complete theoretical model to describe the non-equilibrium fluctuations in the bulk of a ternary mixture at the steady state of a thermodiffusion experiment. 
Keywords: Multicomponent mixtures, Polymer, Thermodiffusion, Shadowgraphy, Nonequilibrium, Fluctuations

\section{Introduction}

The majority of the fluids encountered in nature and industry are multicomponent mixtures. The knowledge of their transport properties is crucial for many different applications from both scientific and technological points of view. The efficiency of exploitation of the crude oil wells, as well as the environmental alternative focused on the $\mathrm{CO}_{2}$ storage in deep brine aquifers, are some recent examples showing the interest of studying the transport properties of multicomponent mixtures subjected to non-equilibrium conditions $[1,2]$. Complex fluids in non-equilibrium conditions exhibit giant fluctuations of the thermodynamic variables, the socalled non-equilibrium fluctuations (NEFs) [3-5]. By analysing NEFs through light scattering techniques, the transport properties of the fluid can be determined both at atmospheric [6] and at high pressure [7].

The non-equilibrium condition can be induced, for example, by applying a temperature gradient to a multicomponent fluid mixture, thus inducing a composition gradient within the fluid by means of the thermodiffusion, or Soret effect [8-10]. This transport process can be investigated by optical techniques and particularly by light scattering thanks to its ability to visualize NEFs without altering the intrinsic properties of the fluid. In this work, dynamic shadowgraphy has been adopted [11-13] to study the fluctuations of the refractive index as generated by the NEFs of the thermodynamic variables, like temperature and concentrations. By shadowgraphy, a large range of fluctuations sizes $\lambda$ or, conversely, wave numbers $q=2 \pi / \lambda$, can be investigated at the same time. This approach, combined with statistical analysis, represents a powerful characterization technique able to provide simultaneous reliable measurements of different 
transport properties, like mass diffusion coefficient or thermal diffusivity as well as Soret coefficient $[6,14,15]$.

A suitable characterization of transport processes in complex mixtures requires a deep understanding of simpler fluids. So far, only binary mixtures $[10,16]$ have been extensively characterized. The extension of theories and experiments from binary to ternary mixtures requires further development due to the intrinsic and significant increase of difficulty with the number of components of the mixture. Currently, a great effort is devoted to investigate the transport phenomena in ternary mixtures, as performed in the present study. Thermodiffusion experiments in ternary mixtures are performed on ground, by using different optical techniques $[10,14,17,18]$, or in microgravity in order to avoid both convection and sedimentation. The thermodynamic characterization of ternary mixtures is one of the objectives of the following ESA projects: Diffusion Coefficient Measurements in ternary mIXtures (DCMIX) [18-23], Soret Coefficients in Crude Oil (SCCO) [1, 24-27] and Giant Fluctuations [28], as further elaborated in a recent colloquium paper [29].

As a benchmark ternary mixture, the tetraline, isobutylbenzene and $n$-dodecane sample has been studied by digital interferometry on ground and in microgravity within the DCMIX experiments $[18,19,30-38]$. In addition, Bataller et al. [17] investigated this mixture on ground by onewavelength shadowgraphy. Since no other alternative was available at that time, Bataller et al. [17] compared their experimental results with the theory of concentration NEFs developed for ternary mixtures in the absence of gravity [39]. The two concentration modes related to the presence of the two independent components could not be distinguished experimentally, and only an average of the two eigenvalues of the mass diffusion matrix could be provided. This result is expected, as the mixture is composed of similar-size molecules, and consequently the two eigenvalues of the diffusion coefficient matrix are very similar to each other. The two eigenvalues $\widehat{D}_{1}$ and $\widehat{D}_{2}$ of the diffusion matrix $\mathbf{D}$ need to be different enough to distinguish the 
two concentration modes. An equivalent way to set this statement is to define a dimensionless number $\operatorname{Dr}=\widehat{D}_{2} / \widehat{D}_{1}\left(\mathrm{Dr}\right.$ for Diffusion eigenvalue Ratio and $\widehat{D}_{1}$ representing the slowest diffusion mode) and require it to satisfy the relation $D r \gg 1$. A ternary mixture containing a polymer dissolved in a binary mixed solvent is a good candidate for such measurement, due to the very different molecular size of its components and, correspondingly, a value of $D r \approx 10$. This was experimentally proved for the diluted polymeric ternary mixture of polystyrenetoluene-n-hexane, as reported in [14], where a layer of $L=2 \mathrm{~mm}$ thick was investigated by using one-wavelength shadowgraphy at a single image acquisition frequency of $28 \mathrm{~Hz}$. By adopting the theoretical model of ternary mixtures in the absence of gravity, the dynamics of the NEFs could be analysed for large wave numbers [14]. From such analysis, it was only possible to determine the two eigenvalues of the mass diffusion matrix and the thermal diffusivity. Afterwards, the diffusion matrix was simplified by neglecting the off-diagonal elements and the result was utilised for obtaining an analytical relationship between the two Soret coefficients, without an independent measurement of them. More recently [40], we have further developed the theory of NEFs to include the effect of gravity, but not confinement so that a direct comparison with the data of Ref. [14] is impossible since fluid layer thickness of only $2 \mathrm{~mm}$ makes confinement effects important, as evidenced in related literature for binary mixtures $[41,42]$.

In the present study, we perform a new shadowgraph experiment on a similar ternary mixture, but in this case a thicker sample with vertical extension of $L=5 \mathrm{~mm}$ is analysed in order to avoid the confinement effect, and be able to compare the experimental results with the theory of NEFs including gravity [40]. The present measurements are also carried out with a faster camera, allowing a more accurate characterization of thermal fluctuations and, thus, a better separation of them from concentration ones. Additionally, a new procedure of image acquisition combined with the concatenation of the structure functions allows us to investigate a wider 
range of correlation times, as required for a complete analysis of this mixture that has a wide range of decay times. The experimental results are thus compared for the first time with the theoretical model developed for ternary mixtures in the presence of gravity [40], as well as with the recently developed theory that includes the coupling of velocity, thermal and solutal NEFs in the case of binary mixtures [43]. These comparisons suggest that a further development of the theory is required, in particular to include the coupling between the different NEFs in the case of the ternary mixture. As part of the contents of this paper we present such a development and compare with the experimental data.

\section{Experimental procedure}

\subsection{Polymeric solution preparation and characterization}

The sample is prepared by dissolving $2 \mathrm{wt} \%$ of polystyrene $\left(\mathrm{PS}, M_{\omega}=4730 \mathrm{~g} / \mathrm{mol}\right.$, DINPoly(styrene) 4730 DA - PDI 1.03 by PSS-polymer) in $49 \mathrm{wt} \%$ toluene (Sigma-Aldrich, 24,451-1, 99.8\%) and 49 wt\% n-hexane (Sigma-Aldrich, 13,938-6, >99\%) at ambient temperature by using a magnetic stirrer. The thermophysical properties of the mixture, such as density, viscosity and both thermal and mass expansion coefficients are determined.

The kinematic viscosity $v$ is measured at $25^{\circ} \mathrm{C}$ by a capillary viscometer (Ubbelohde SCHOTT). The thermal and mass expansion coefficients, $\alpha$ and $\beta_{i}$, respectively, are defined as follows:

$$
\begin{aligned}
& \alpha=-\frac{1}{\rho}\left(\frac{\partial \rho}{\partial T}\right)_{\omega, p}, \\
& \beta_{i}=\frac{1}{\rho}\left(\frac{\partial \rho}{\partial \omega_{i}}\right)_{T, p},
\end{aligned}
$$

where $\rho$ is the mixture density, $T$ is the temperature and $\omega_{i}$ is the concentration of component $i$ in mass fraction. Following a common notation, components are ordered according to their 
(pure) density, hence, in this study 1 corresponds to PS, 2 corresponds to toluene and 3 corresponds to n-hexane.

The coefficients $\alpha$ and $\beta_{i}$ are indirectly determined from measurements of the mixture density performed at different conditions through a Density Meter (ANTON PAAR, DMA 5000). For the thermal expansion coefficient, the density is measured at different temperatures (from 23 to $27^{\circ} \mathrm{C}$ ), while keeping the concentrations constant at $\omega_{1}=0.02$ and $\omega_{2}=0.49$. The mass expansion coefficients are obtained after measuring the density at different concentrations while keeping the temperature constant at $25^{\circ} \mathrm{C}$. To obtain the mass expansion coefficient of the polymer in the mixed solvent, $\beta_{1}$, the concentration of the polystyrene is changed from $\omega_{1}=$ 0.000 to $\omega_{1}=0.035$, keeping $\omega_{2}=\omega_{3}$. For the mass expansion coefficient of the mixed solvent, $\beta_{2}$, the composition of the mixed solvent is modified from $\omega_{2}=0.47$ to $\omega_{2}=0.51$, keeping $\omega_{1}=0.02$ constant. Measured values of $\rho, v, \alpha$ and $\beta_{i}$ are summarized in Table 1 . The values of $\rho, v, \alpha$ and $\beta_{2}$ are consistent with those reported in [44], determined for the equimassic toluene-n-hexane binary mixture at $T_{\text {mean }}=25^{\circ} \mathrm{C}$. The measurements of viscosity and density are repeated at least three times for each sample. The values reported in Table 1 are calculated as the average of the three measurements and the corresponding uncertainties are the standard deviations. The parameter $\alpha$ is determined through Eq. 1, i.e. dividing the slope of the density vs. temperature by the density of the sample at the nominal temperature. Similarly, $\beta_{i}$ are calculated through Eq. 2, i.e. dividing the slope of the density vs. concentration by the density of the sample at the nominal concentration.

\section{Table 1: Thermophysical properties of the polystyrene-toluene-n-hexane mixture}

\begin{tabular}{|c|c|}
\hline Parameter & Value \\
\hline$\rho$ & $(750.0 \pm 0.4) \times 10^{-3} \mathrm{~g} / \mathrm{cm}^{3}$ \\
\hline$v$ & $(5.24 \pm 0.01) \times 10^{-3} \mathrm{~cm}^{2} / \mathrm{s}$ \\
\hline
\end{tabular}




\begin{tabular}{|c|c|}
\hline$\alpha$ & $(9.1 \pm 0.7) \times 10^{-4} / \mathrm{K}$ \\
\hline$\beta_{1}$ & $(3.1 \pm 0.1) \times 10^{-1}$ \\
\hline$\beta_{2}$ & $(2.6 \pm 0.1) \times 10^{-1}$ \\
\hline
\end{tabular}

\subsection{Thermodiffusion experiment}

The experimental set-up is quite similar to the one used in previous studies and a more detailed description can be found elsewhere $[6,7]$. Before filling our thermodiffusion cell, its interior is evacuated by using a vacuum pump for more than four hours in order to evaporate all the residual fluids. Subsequently, the mixture is injected into the cell. The sample is sandwiched between two square sapphire windows of $8 \mathrm{~mm}$ thick, mechanically separated to provide a vertical thickness of the fluid layer of $L=(5.0 \pm 0.1) \mathrm{mm}$. Two thermoelectric (TEC) Peltier devices are used to apply the temperature gradient to the fluid mixture. Both TECs are independently connected to two proportional-integral-derivative (PID) temperature controllers (Wavelength Electronics, MODEL LFI - 3751), being able to set the temperatures of the two sides of the fluid slab with an absolute accuracy of $0.01 \mathrm{~K}$ and a relative RMS stability of $1 \mathrm{mK}$ over 24 hours. The temperature gradient is applied in the $z$ direction, and the sample is always heated from above in order to avoid convection, since both Soret coefficients are expected to be positive [44-46]. When applying a temperature gradient, the two TECs actually act as heat pumps in the same direction, thus a heat exchanger flushed with water coming from a thermostat (HUBER, ministate 125) removes the excess heat from the external side of the Peltier elements. The thermodiffusion experiments are performed by imposing temperature differences of $\Delta T=$ 10,15 and $20 \mathrm{~K}$ over the vertical extension of the sample cell. After imposing the temperature gradient, and before starting the image acquisition through the shadowgraph setup, it is necessary that the sample reaches the stationary state where the Soret effect is completely balanced by Fickean diffusion. In our experimental conditions, the diffusion time $\tau=L^{2} / D$ is 
of the order of $8.3 \times 10^{4} \mathrm{~s}$, i.e. about 23 hours. This value is obtained from the diffusion coefficient of the polymer in a typical solvent, like toluene $D \approx 3 \times 10^{-6} \mathrm{~cm}^{2} / \mathrm{s}$ [45].

Density fluctuations $\delta \rho$ generated by both temperature fluctuations $\delta T$ and concentration fluctuations $\delta \omega_{i}$ give rise to refractive index fluctuations $\delta n=\delta T(\partial n / \partial T)+$ $\sum_{i} \delta \omega_{i}\left(\partial n / \partial \omega_{i}\right)$, which can be detected by optical techniques, as described in the literature $[11,13,47]$. In general, velocity fluctuations are not supposed to induce fluctuations of the density or of the refractive index.

In our optical setup, a super-luminous diode (Superlum, SLD-MS-261-MP2-SM) with a wavelength of $\lambda=(675 \pm 13) \mathrm{nm}$ acts as the light source, illuminating the sample parallel to the temperature gradient. The light is provided out of a monomode optical fibre and the beam is collimated by an achromatic doublet lens with focal length $f=150 \mathrm{~mm}$ and placed at its focal distance from the fibre output. After the collimating lens, a linear polariser is positioned before the sample and a second linear polariser (analyser) after it, providing a control of the light intensity independent of the integration time of the detector. Finally, the light beam impinges onto a scientific-CMOS camera (Hamamatsu Digital Camera C13440, ORCA - Flash 4.0) whose detector size is $s=1.33 \mathrm{~cm}$, placed in the near field, i.e. at a distance $z=$ $(200 \pm 5) \mathrm{mm}$ from the sample midplane. The s-CMOS camera allows a 'fast' image acquisition frequency up to $100 \mathrm{~Hz}$ at full frame $(2048 \times 2048$ pixels $)$, thus enabling a fruitful characterization of the temperature fluctuations in the observable wave number range. The shadowgraph signal is the result of the interference of the transmitted beam with the ones scattered by the sample refractive index fluctuations. In our experiment series of $N=2500$ images of $2048 \times 2048$ pixels, corresponding to $q_{\min }=2 \pi / \mathrm{s}=4.72 / \mathrm{cm}$, are recorded for three different frequencies of $f=100,10,1 \mathrm{~Hz}$, corresponding to minimum correlation times of $d t_{\min }=0.01,0.1,1 \mathrm{~s}$. The recorded images are eventually analysed by the Differential Dynamic Algorithm (DDA) briefly described in the following section [48]. 


\subsection{Dynamic shadowgraphy}

196

197

198

199

200

201

202

203

204

205

206

207

208

209

210

211

212

213

The DDA analysis consists of the following steps: images are recorded at different times as bidimensional intensity maps $I(\vec{x}, t)$ and are 2D-Fourier transformed and normalized to get $i(\vec{q}, t)=I(\vec{q}, t) / I(0, t)$. Then, differences between pairs of normalised Fast Fourier Transforms (FFTs) are calculated and their square moduli are determined $|\Delta i(\vec{q}, t, d t)|^{2}=$ $|i(\vec{q}, t)-i(\vec{q}, t+d t)|^{2}$. These quantities are computed for all possible delay times $d t$, multiples of the $d t_{\min }$ set by the acquisition frequency of the detector. Finally, the results are averaged first over time $\left\langle|\Delta i(\vec{q}, d t)|^{2}\right\rangle=\left\langle|\Delta i(\vec{q}, t, d t)|^{2}\right\rangle_{t}$ and second over the modulus of the wave vector $\vec{q},\left\langle|\Delta i(q, d t)|^{2}\right\rangle=\left\langle|\Delta i(\vec{q}, d t)|^{2}\right\rangle_{|\vec{q}|}$. The resultant function is the so-called Structure Function $(\mathrm{SF})\left\langle|\Delta i(q, d t)|^{2}\right\rangle$. All these calculations are performed by a custom software running on a graphic card and taking advantage of the massive parallelization of the Graphic Processing Unit (GPU), thus requiring about one hour of computational time for each experiment, i.e. the three series of 2500 images of $2048 \times 2048$ pixels [49].

\subsection{Concatenation of the experimental structure function}

As mentioned above, in the experiments reported in this study, sets of images of $2048 \times 2048$ pixels have been recorded at three different acquisition frequencies at the steady state of the thermodiffusion process. Then, the experimental SF is determined by analysing each stack of images acquired at a specific frequency. Examples of $\left\langle|\Delta i(q, d t)|^{2}\right\rangle$ are shown in Fig. 1, as a function of the wave number (Fig. 1a, 1c, 1e) and as a function of the correlation time $d t$ for different wave numbers (Fig. 1b, 1d, 1f). The SFs obtained at three different frequencies are then merged in order to form a single concatenated SF, or c-SF (Fig. 1g), that covers a large range of correlation times of about four orders of magnitude, as shown in Fig. 1h. 
As stated above, the c-SF is the result of merging the SFs determined at three different

219 frequencies (from left to right in Fig. 1h): the first points correspond to the data acquired at 100 $\mathrm{Hz}$ (from Fig. 1b, dark purple squares at short $d t$ ), the second ones to $10 \mathrm{~Hz}$ (from Fig. 1d, light cyan circles at intermediate $d t$ ) and the third ones to $1 \mathrm{~Hz}$ (from Fig. 1f, dark orange triangles

222 at large $d t$ ). The concatenation procedure is made by simply selecting the relevant part of the 223 SFs as shown in Fig. 1. About the $10 \%$ of the delay times of each data set are used for the 224 concatenation, disregarding those that are already included in the slower frequency slot. This means that from the fastest acquisition set only delay times in the range $0.01-2.5 \mathrm{~s}$ are taken, from the intermediate one the selected range is $2.5-25 \mathrm{~s}$ and from the slowest one $25-250 \mathrm{~s}$. The 227 concatenation procedure has been already applied in our precedent work [43], but here it is 228 described in more detail. We point out that this scheme becomes useful whenever it is necessary to investigate NEFs with quite different decay times and that it is similar to the multi-tau approach utilised in dynamic light scattering [50]. 

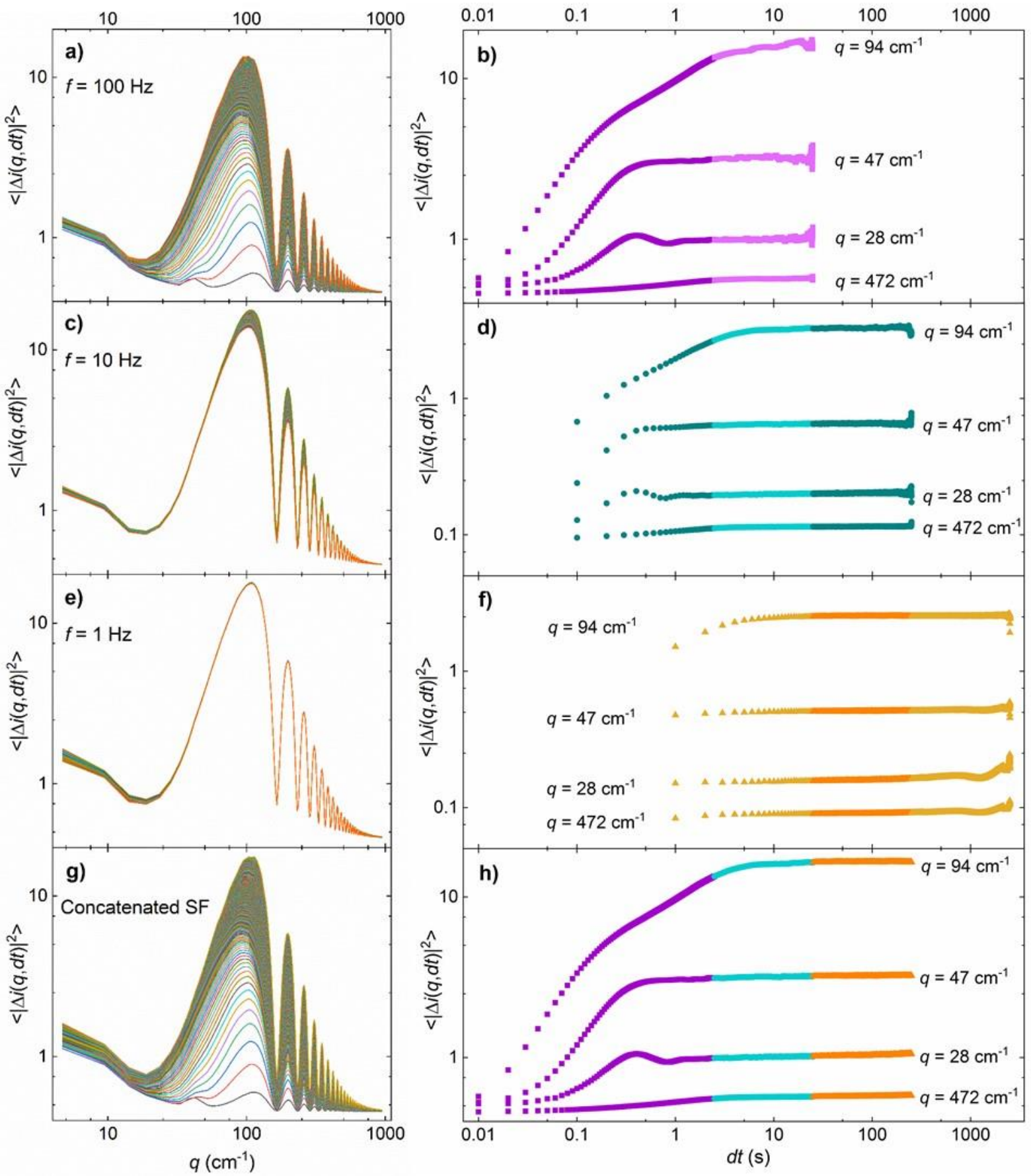

Figure 1: Structure function of the thermodiffusion experiment carried out at temperature difference of $20 \mathrm{~K}$ for a series of images of $2048 \times 2048$ pixels at 100, 10 and $1 \mathrm{~Hz}$, as a function of the wave number (a, $c$, e, respectively) and as a function of correlation time (b, $d, f$, respectively). Concatenated structure function as a function of the wave number (g) and as a function of time (h). Purple squares, cyan circles and orange triangles correspond to the data acquired at 100, 10 and $1 \mathrm{~Hz}$, respectively. 


\subsection{Analysis of experimental signals}

After the DDA and the concatenation process one gets the c-SF; it represents the raw experimental signals, obtained only from the acquired image series. For the analysis of these concatenated $\left\langle|\Delta i(q, d t)|^{2}\right\rangle$, the physical optics theory of shadowgraph proposes the following expression $[12,48]$ :

$$
\left\langle|\Delta i(q, d t)|^{2}\right\rangle=2\left\{T(q) S(q)\left[1-I_{S F}(q, d t)\right]+B(q)\right\}
$$

where $T(q)$ is the shadowgraph optical transfer function [11], $S(q)$ is the static power spectrum, $B(q)$ is the background and $I_{S F}(q, d t)$ is the intermediate scattering function, that usually is described as a sum of exponential decays: $I_{S F}(q, d t)=\sum_{i} a_{i} \exp \left(-d t / \tau_{i}(q)\right)$, where $a_{i}$ are the amplitudes of the different modes (with $\sum_{i} a_{i}=1$ ) and $\tau_{i}(q)$ the wave-number dependent relaxation times.

A simple observation of Fig. 1h, without any calculation, shows that, depending on the wave number range, up to three different decay times can be distinguished in the c-SF. In some cases, these decay times differ by more than one order of magnitude. In addition, and also depending on the wave number range, one can observe in Fig. 1h damped oscillations in $\left\langle|\Delta i(q, d t)|^{2}\right\rangle$. This oscillating (propagating) phenomenon means that at least two of the relaxation times $\tau_{i}(q)$ present in the $I_{S F}(q, d t)$ of Eq. 3 (and their associated amplitudes) form a pair of complex conjugate numbers. We conclude that a complex scenario of NEFs with different decay times is present in the case of a ternary mixture with large $L e$ and $\mathrm{Dr}$ numbers, and a key question is the selection of the number of modes in the $I_{S F}(q, d t)$ of Eq. 3 and whether they are purely diffusive (monotonically decaying) or propagating (oscillating). A rational choice needs to be guided by the available theoretical models for NEFs in ternary mixtures, which we review next.

\section{Theoretical framework}




\subsection{Thermodiffusion in ternary mixtures}

In a ternary mixture, the mass concentrations of the three components are related by: $\omega_{1}+$ $\omega_{2}+\omega_{3}=1$, which means that only two concentrations, e.g. $\omega_{1}$ and $\omega_{2}$, are independent. The corresponding diffusion fluxes $J_{1}$ and $J_{2}$ in the centre-of-mass frame of reference have thermodiffusion and Fickean components, as expressed by [51]:

$$
\left(\begin{array}{l}
J_{1} \\
J_{2}
\end{array}\right)=-\rho\left[\left(\begin{array}{ll}
D_{11} & D_{12} \\
D_{21} & D_{22}
\end{array}\right)\left(\begin{array}{l}
\nabla \omega_{1} \\
\nabla \omega_{2}
\end{array}\right)+\left(\begin{array}{cc}
\omega_{1}\left(1-\omega_{1}\right) & -\omega_{1} \omega_{2} \\
-\omega_{1} \omega_{2} & \omega_{2}\left(1-\omega_{2}\right)
\end{array}\right)\left(\begin{array}{l}
\mathrm{D}_{T 1} \\
\mathrm{D}_{T 2}
\end{array}\right) \nabla T\right],
$$

where $\rho$ is the density of the mixture, $D_{i j}$ are the components of the centre-of-mass diffusion matrix, $\nabla \omega_{i}$ are the concentration gradients, $D_{T_{i}}$ are the invariant thermodiffusion coefficients and $\nabla T$ is the applied temperature gradient.

At the stationary state, the diffusion fluxes become zero and, following Ortiz de Zárate [51], the Soret coefficients $S_{T_{i}}$ can be expressed by:

$$
\left(\begin{array}{cc}
\omega_{1}\left(1-\omega_{1}\right) & -\omega_{1} \omega_{2} \\
-\omega_{1} \omega_{2} & \omega_{2}\left(1-\omega_{2}\right)
\end{array}\right)\left(\begin{array}{l}
S_{T 1} \\
S_{T 2}
\end{array}\right) \nabla T=-\left(\begin{array}{l}
\nabla \omega_{1} \\
\nabla \omega_{2}
\end{array}\right)
$$

The introduction of the matrix concentration pre-factors in Eqs. 4 and 5 forces the thermodiffusion coefficients $D_{T_{i}}$ and the Soret coefficients $S_{T_{i}}$ to display the same frameinvariance properties of the single $D_{T}$ or $S_{T}$ of a binary mixture, that is, they are numerically the same irrespective of whether mass fractions or mole fractions are used to express the composition [51]. Here, for simplicity, we display the equations only in the mass fraction framework.

Fluctuating Hydrodynamics (FHD) has been recently extended to ternary mixtures first considering equilibrium fluctuations [52] and then non-equilibrium fluctuations $[39,40]$. The first NE theory [39] neglected the effects of gravity and confinement. In that case, there is no mixing between the decay rates of the four hydrodynamic modes (fluctuations of velocity, temperature and two concentrations) which continue to decay with their well-known equilibrium values given by kinematic viscosity, thermal diffusivity and the two eigenvalues 
$\widehat{D}_{i}$ of the diffusion matrix $\mathbf{D}$. These $\widehat{D}_{i}$, are always real and positive, and can be related to the four components $D_{i j}$ of Eq. 4 by $[39,40]$ :

$$
\widehat{D}_{1,2}=\frac{1}{2}\left[D_{11}+D_{22} \mp \sqrt{\left(D_{11}-D_{22}\right)^{2}-4 D_{12} D_{21}}\right] .
$$

In accordance with the aforementioned common notation (i.e. 1 for PS, 2 for toluene and 3 for n-hexane), $\widehat{D}_{1}<\widehat{D}_{2}$ since $\widehat{D}_{1}$ represents the slowest diffusion mode essentially related to the diffusion of the polystyrene in the binary solvent.

Since refractive index does not depend on fluid velocity, only three of the four hydrodynamic modes are directly observable by optical techniques. Hence, within the approximations of Ref. [39] (no gravity and no confinement) we conclude that for a thermodiffusion experiment in a ternary mixture, a suitable expression for the $I_{S F}(q, d t)$ can be provided by the sum of three exponential decays given by two concentration modes, plus one mode for temperature fluctuations:

$I_{S F}(q, d t)=a_{1} \exp \left[-d t / \tau_{1}(q)\right]+a_{2} \exp \left[-d t / \tau_{2}(q)\right]+\left(1-a_{1}-a_{2}\right) \exp \left[-d t / \tau_{3}(q)\right]$

where $a_{i}$ are the normalised amplitudes of the different modes and $\tau_{i}(q)$ the corresponding relaxation times.

\subsection{Concentration NEFs in ternary mixtures including gravity effects}

For NEFs, first the theory was developed in the absence of gravity force [39]; while gravity effects were incorporated by Martínez Pancorbo et al. [40]. Both of these papers adopted the so-called large Lewis number approximation that uncouples temperature fluctuations from concentration fluctuations. Next, we briefly summarize the main results of Ref. [40] and recall the expressions useful for the experimental data analysis. 
The presence of gravity causes a mix between the two concentrations NEFs. The corresponding decay times can be described as $\tau_{i}(q)=1 /\left[\gamma_{i}(q) q^{2}\right]$, where the diffusivities $\gamma_{i}(q)$ in dimensionless form, are given by the following equation [40]:

$$
\gamma_{i}(\tilde{q})=\frac{1}{2}\left(\widehat{D}_{1}+\widehat{D}_{2}-\frac{R a_{i} \widehat{D}_{i}}{\tilde{q}^{4}}\right) \times\left[1 \mp \sqrt{1-\frac{\frac{4 \widehat{D}_{1} \widehat{D}_{2}}{\left(\widehat{D}_{1}+\widehat{D}_{2}\right)^{2}}\left[1-\frac{R a_{\text {S,ter }}}{\widetilde{q}^{4}}\right]}{\left[1-\frac{R a_{i} \widehat{D}_{i}}{\tilde{q}^{4}\left(\widehat{D}_{1}+\widehat{D}_{2}\right)}\right]^{2}}}\right],
$$

where $\tilde{q}=q L$ is the dimensionless wave number, and $R a_{i}$ are the two solutal Rayleigh numbers defined for ternary mixtures by:

$$
\begin{aligned}
& R a_{i}=\frac{g L^{4}}{v \widehat{D}_{i}} \beta_{i}^{\prime} \nabla \omega_{i}^{\prime}, \\
& R a_{i} \widehat{D}_{i}=R a_{1} \widehat{D}_{1}+R a_{2} \widehat{D}_{2}, \\
& R a_{S, t e r}=R a_{1}+R a_{2},
\end{aligned}
$$

In Eq. 9, the primes indicate the concentrations (mass fractions) in which the diffusion matrix is diagonal, for a generic diffusion matrix [53]:

$$
\left(\begin{array}{c}
\omega_{1}^{\prime} \\
\omega_{2}^{\prime}
\end{array}\right)=\left(\begin{array}{cc}
1 & \frac{D_{22}-\widehat{D}_{2}}{D_{21}} \\
\frac{D_{11}-\widehat{D}_{1}}{D_{12}} & 1
\end{array}\right)\left(\begin{array}{l}
\omega_{1} \\
\omega_{2}
\end{array}\right) .
$$

The use of "diagonal" concentrations simplifies the theory but, as we shall see, somewhat complicates the interpretation of experimental results. In general, they differ from the measurable mass fractions.

In summary, in this model [40] the two concentration modes couple, while temperature fluctuations remain uncoupled, keeping their equilibrium decay rate unaltered. Since for typical values of $R a_{i}$ the two decay times of Eq. 8 are real numbers, this theory [40] suggests to still use Eq. 7 for the $I_{S F}(q, d t)$. We note that the notation of Eqs. 9-11 implies that, for a ternary mixture, two Lewis numbers $\left(L e_{1}\right.$ and $\left.L e_{2}\right)$ can be defined as $L e_{i}=a_{T} / \widehat{D}_{i}$.

\subsection{Coupling of concentration and temperature NEFs in binary mixtures}


In the simplest case of binary mixtures, most of the available literature on concentration and temperature NEFs makes use of the simplification that the two modes are always decoupled. This is essentially true whenever the large Lewis number approximation is valid, that is the case of ordinary liquid mixtures whose mass diffusion coefficient is much smaller than the thermal diffusivity. However, the decoupling assumption fails to be valid for very large fluctuations, i.e. for small wave numbers, as recently demonstrated both experimentally and theoretically [43]. In particular, only the full coupling of velocity, temperature and concentration NEFs in the bulk fluid can explain the appearance of propagating modes giving rise to a $I_{S F}(q, d t)$ that includes oscillations at very small wave numbers. This mechanism also induces a slowing-down of the non-propagating concentration NEFs similar to that caused by the confinement effect $[41,42]$.

This theory of full coupling between NE velocity, temperature and concentrations fluctuations has been published only for binary mixtures. We briefly recall here the main steps of the theoretical framework, while for details we address the reader to the literature [43].

In order to take into account a more complete description of the behaviour of NEFs within a thermodiffusion experiment, one should write the evolution equations of the fluctuations of velocity $v_{z}$, temperature and concentration of the single independent component:

$$
\frac{\partial}{\partial t}\left[\begin{array}{c}
\delta v_{z}(q, t) \\
\delta T(q, t) \\
\delta \omega(q, t)
\end{array}\right]=-\mathbf{G}(q)\left[\begin{array}{c}
\delta v_{z}(q, t) \\
\delta T(q, t) \\
\delta \omega(q, t)
\end{array}\right],
$$

where the inverse linear response matrix in its dimensionless form can be written as:

$$
\mathbf{G}(\tilde{q})=\left[\begin{array}{ccc}
\operatorname{Pr} L e \tilde{q}^{2} & -1 & 1 \\
-\operatorname{Ra} a_{s} \operatorname{PrLe} / \psi & L e \tilde{q}^{2} & 0 \\
R a_{s} \operatorname{PrLe} & \psi \tilde{q}^{2} & \tilde{q}^{2}
\end{array}\right]
$$

In the previous equation the well-known dimensionless numbers appear, namely the Lewis number $L e=a_{T} / D$, where $a_{T}$ is the thermal diffusivity; the Prandtl number $\operatorname{Pr}=v / a_{T}$; the 


$$
\frac{\partial}{\partial t}\left[\begin{array}{c}
\delta v_{z}(q, t) \\
\delta T(q, t) \\
\delta \omega_{1}(q, t) \\
\delta \omega_{2}(q, t)
\end{array}\right]=-\mathbf{G}_{t}(q)\left[\begin{array}{c}
\delta v_{z}(q, t) \\
\delta T(q, t) \\
\delta \omega_{1}(q, t) \\
\delta \omega_{2}(q, t)
\end{array}\right],
$$

375 where the inverse linear response matrix for "diagonal" concentrations can be written in

separation ratio $\psi=c_{0}\left(1-c_{0}\right) S_{T} \beta / \alpha$; and the solutal Rayleigh number $R a_{S}=$ $[\beta g \nabla \omega /(\nu D)] L^{4}$

The solution of Eqs. 13 and 14 requires the computation of the eigenvalues and eigenvectors of the matrix $\mathbf{G}(\tilde{q})$ :

$$
\operatorname{det}[\mathbf{G}(\tilde{q})-\lambda \mathbf{1}]=\left[\Gamma_{v_{z}}(\tilde{q})-\lambda\right]\left[\Gamma_{T}(\tilde{q})-\lambda\right]\left[\Gamma_{\omega}(\tilde{q})-\lambda\right]
$$

with NE fluctuations dimensionless decay times given by $\tau_{i}(\tilde{q})=1 /\left[\Gamma_{i}(\tilde{q})\right]$. Although Eq. 15 can be solved analytically, the resulting expressions of the roots as a function of $q$ are so complicated that, in practice, it is more useful to have a numerical solution for fixed values of the parameters $\operatorname{Pr}, L e, R a_{S}$ and $\psi$, in the whole range of $q$.

Depending on the parameter values and on the wave number $q$, Eq. 15 may have a pair of complex conjugate solutions, which typically happens at small $q$ values. The resulting $I_{S F}(q, d t)$ function in those cases shows a sinusoidal term:

$$
I_{S F}(q, d t)=a_{1} \exp \left[-d t / \tau_{1}(q)\right]+\left\{\frac{1-a_{1}}{\cos [\phi(q)]}\right\} \cos [\Omega(q) d t+\phi(q)] \exp \left[-d t / \tau_{2}(q)\right]
$$

where $\Omega(q)$ is the oscillation frequency and $\phi(q)$ a phase term.

\subsection{Coupling of concentration and temperature NEFs in ternary mixtures}

The next step in the development of FHD is to include all the couplings among the fluctuating modes for a ternary mixture. Of course, in that case one needs to consider the evolution of fluctuations in velocity $\delta v_{z}$, temperature and concentration of the two independent components: dimensionless form as: 


$$
\mathbf{G}_{\boldsymbol{t}}(\tilde{q})=\left[\begin{array}{cccc}
\operatorname{Pr} L e_{t} \tilde{q}^{2} & -1 & 1 & 1 \\
-\operatorname{Pr} L e_{t}{ }^{2} R a & L e_{t} \tilde{q}^{2} & 0 & 0 \\
\operatorname{Pr} L e_{t}{ }^{2} R a \psi_{1}^{\prime} & \psi_{1}^{\prime} \tilde{q}^{2} & \tilde{q}^{2} & 0 \\
\operatorname{Pr} L e_{t}{ }^{2} R a \psi_{2}^{\prime} & \operatorname{Dr} \psi_{2}^{\prime} \tilde{q}^{2} & 0 & \operatorname{Dr} \tilde{q}^{2}
\end{array}\right]
$$

To make dimensionless Eqs. 17 and $18, L$ is used as unit of length and $L^{2} / \widehat{D}_{1}$ as unit of time; consequently, $\tilde{q}=q L$ for wave numbers, and $L / \widehat{D}_{1}$ is the unit of velocity. Hence, everything is made dimensionless with respect to the slower diffusive mode. Other dimensionless parameters in Eq. 18 are: one (unique) ternary Lewis number $L e_{t}=a_{T} / \widehat{D}_{1}$, the Diffusion eigenvalue Ratio $\operatorname{Dr}=\widehat{D}_{2} / \widehat{D}_{1}$, the thermal Rayleigh number $R a=-\alpha g L^{4} \nabla T /\left(v a_{T}\right)$ and the "diagonal" separation ratios $\psi_{i}^{\prime}=D_{T, i}^{\omega \prime} \beta_{i}^{\prime} /\left(\widehat{D}_{i} \alpha\right)$, with $D_{T, i}^{\omega \prime}$ being the (diagonal) thermodiffusion coefficients as defined in Ref. [18]. We point out that this approach is different from the one used in our previous publication [40] and reported in section 3.2, where two Lewis numbers were introduced.

As in the case of binary mixtures, the solution of Eq. 17 requires to numerically evaluate the dimensionless decay rates $\Gamma_{i}(\tilde{q})$ as the four eigenvalues of the matrix $\mathbf{G}_{\boldsymbol{t}}(\tilde{q})$, namely:

$$
\operatorname{det}\left[\mathbf{G}_{\boldsymbol{t}}(\tilde{q})-\lambda \mathbf{1}\right]=\left[\Gamma_{\nu_{z}}(\tilde{q})-\lambda\right]\left[\Gamma_{T}(\tilde{q})-\lambda\right]\left[\Gamma_{\omega_{1}}(\tilde{q})-\lambda\right]\left[\Gamma_{\omega_{2}}(\tilde{q})-\lambda\right] .
$$

A more detailed explanation of the full-coupling theory for ternary mixtures is outside the scope of the present publication and will be the subject of a future paper. We simply mention that, depending on $q$, complex solutions of Eq. 19 may exist. As was the case with binaries, for parameter values typical of ternary liquid mixtures, these propagating modes do appear as a mixing between the temperature and the viscous (velocity) modes. The consequence is an oscillatory $I_{S F}(q, d t)$ such as the one expressed in Eq. 16, but with an additional exponential term to represent the extra concentration mode.

\section{Results}




\subsection{Intermediate scattering function analysis}

As mentioned in section 2.5, for most wave numbers the $I_{S F}(q, d t)$ of Eq. 3 is usually defined as a sum of exponential decays. However, a preliminary analysis of the experimental SF is required to check the suitability of this model. As it can be seen in the c-SF shown in Fig. 1h for $q=94 / \mathrm{cm}$, three exponential decays can be detected, corresponding (from left to right) to the relaxation of the temperature and concentration (faster and slower mass modes) NEFs. Then, the $I_{S F}(q, d t)$ can be modelled for most wave numbers by a sum of three exponential decays, as defined in section 3.1 by Eq. 7 for a system including one thermal mode plus two solutal, well separated, modes. In the studied system, the corresponding three exponential decays represent the following three modes: long decay times for the two concentration modes, slower for mass diffusion of the denser component (PS in the binary solvent), and faster for mass diffusion of the two components of the solvent mixture (toluene and n-hexane); and the shortest decay time corresponds to the thermal diffusivity of the overall mixture.

Nevertheless, for small wave numbers such as $q=28 / \mathrm{cm}$, oscillations are detected in the cSF (see Fig. 1h), indicating the presence of propagating modes [43]. Fig. 2 shows the oscillations in the experimental c-SF for several wave numbers in the range $19 / \mathrm{cm}<q<$ $47 / \mathrm{cm}$. These need to be taken into account by using a proper $I_{S F}(q, d t)$ function, such as the one described in section 3.3 by Eq. 16. The latter equation is defined for a binary mixture, and includes one solutal mode, i.e. the exponential decay, and a coupled thermo-viscous mode, i.e. the damped oscillation. For analysing ternary mixtures, one should supplement Eq. 16 with an additional exponential term, representing the second solutal mode. However, after carrying out a careful comparison of the corresponding fitting output, we have found that the addition of this term gives more scattered results due to the increase in the number of fitting parameters. For that reason, Eq. 16 has been used to fit the raw data in the wave number range where oscillations 
are detected in the c-SF. The slower concentration mode is, thus, unobservable in this wave number range.

426

427

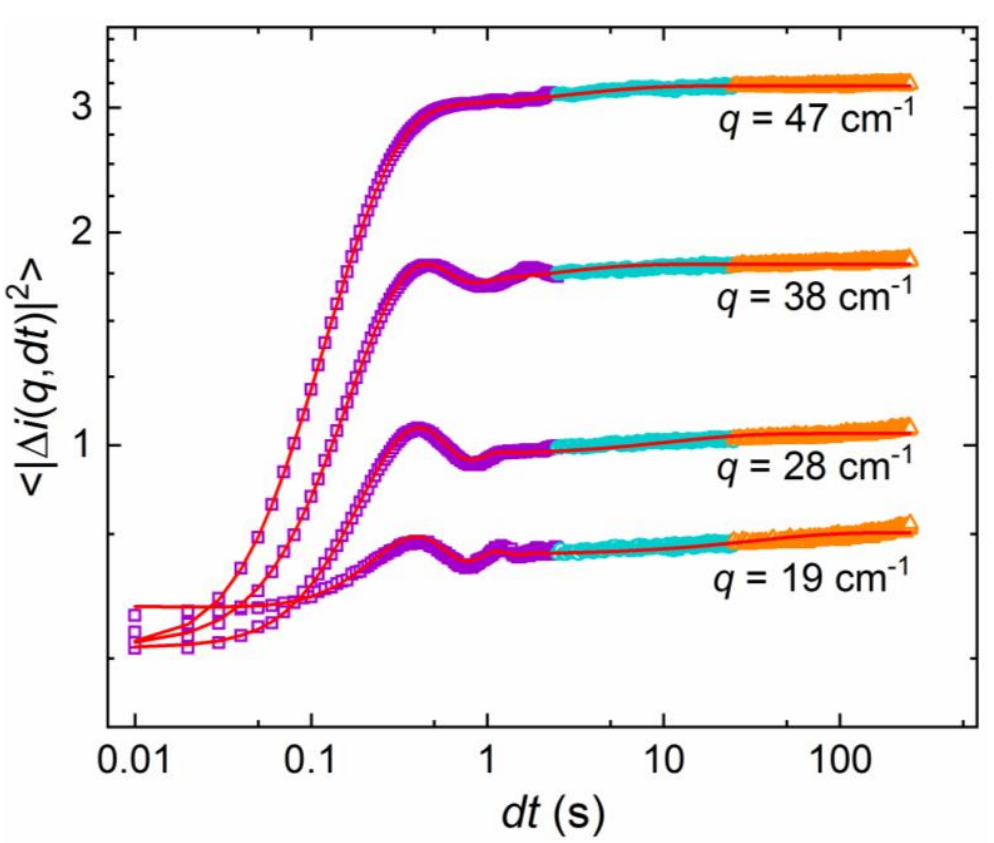

Figure 2: Concatenated structure function of the thermodiffusion experiment carried out at temperature difference of $20 \mathrm{~K}$ for a series of images of $2048 \times 2048$ as a function of time for different small wave numbers. Purple squares, cyan circles and orange triangles correspond to the data acquired at 100, 10 and $1 \mathrm{~Hz}$, respectively, and the red curves to the fitting functions through Eqs. 3 and 16.

Generally, the quality of these fittings is good, as demonstrated in Fig. 2, with an average $R^{2}$ of about 0.99 , which translates into a $3 \%$ mean relative uncertainty for the decay times determined by these fittings. However, both $R^{2}$ and the relative uncertainty of the decay times depend on the wave number. The best accuracy is obtained for intermediate wave numbers where the signal-to-noise ratio of the c-SF is the largest (see Fig. 1g). Decay times with a relative uncertainty larger than $10 \%$ have been disregarded for the further analysis. 
442 Figure 3 shows the decay times of the different fluctuating modes as resulting from the 443 modelling of the experimental c-SF by using Eqs. 3 and Eq. 7 or Eq. 16 depending on the

444 analysed $q$. Three decay times are easily distinguished (from top to bottom): the slowest mode (black symbols) describes the concentration mode of the polymer into the binary solvent; the intermediate one (blue symbols) the concentration mode of the binary solvent mixture; and the fastest one (red symbols) the temperature mode. These decay times cover a span of about three orders of magnitude, which is quite impressive for an imaging technique. This justifies the necessity of performing measurements at different acquisition frequencies and of carrying out the concatenation procedure adopted here. Besides decay times, when the Eq. 16 is used, the fitting procedure also gives the oscillation frequency $\Omega(q)$ of the propagating modes appearing in Fig. 2 for wave numbers $q<47 / \mathrm{cm}$.

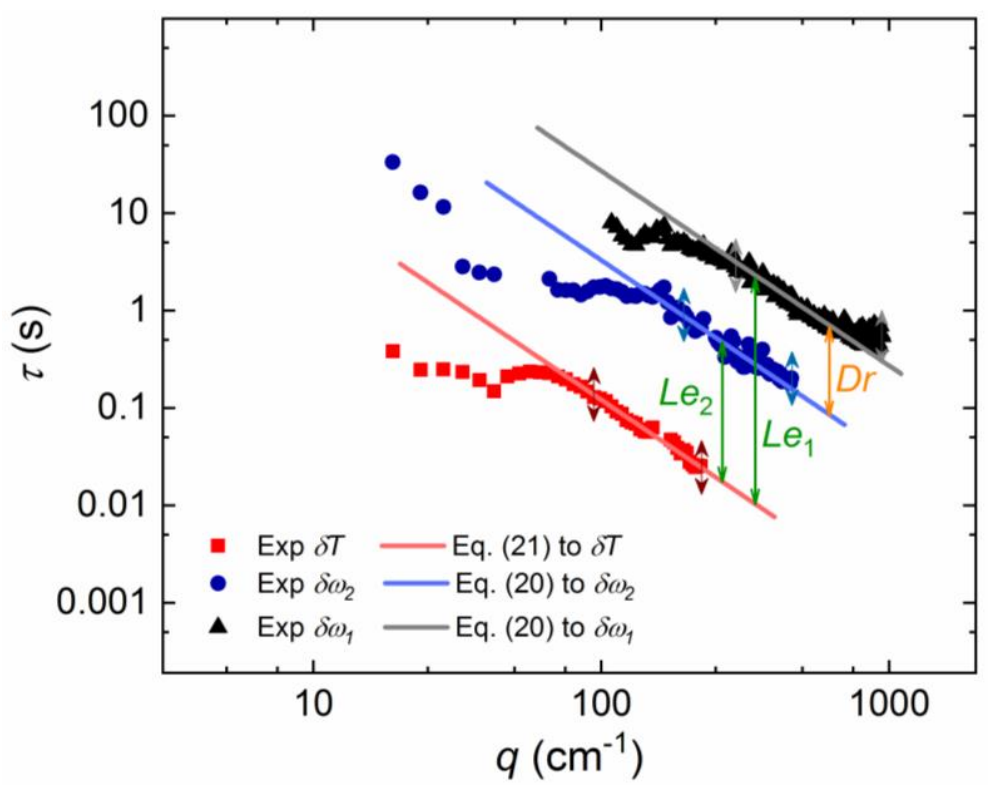

Figure 3: Decay times of NEFs on the ternary mixture of PS-toluene-n-hexane in a thermodiffusion experiment performed at temperature difference of $20 \mathrm{~K}$, as a function of the wave number q. Lines represent fitting functions through Eqs. 20-21 over the diffusive wave number range, as indicated in the text. 
The experimental decay times shown in Fig. 3 are to be discussed first in the diffusive regime only (for large enough wave numbers), where the three decay times are well-separated. As elucidated at the end of section 3.1, in this region of large wave numbers the effect of gravity is negligible for both concentration modes, and the decay times of the two concentration modes are given by the eigenvalues of the diffusion matrix:

$$
\tau_{i}(q)=1 /\left(\widehat{D}_{i} q^{2}\right)
$$

By fitting the experimental decay times in the diffusive regime $(q>290 / \mathrm{cm}$ for the concentration NEFs, c-NEFs, of component 1 and $q>190 / \mathrm{cm}$ for the c-NEFs of component 2) to Eq. 20, the eigenvalues of the diffusion matrix can be determined. The diffusion coefficient $\widehat{D}_{1}$ obtained from the slowest concentration mode represents the mass diffusion eigenvalue of the polymer in the binary solvent mixture. $\widehat{D}_{2}$ calculated from the intermediate decay times (i.e. faster concentration mode) represents the mass diffusion eigenvalue of toluene in n-hexane (i.e. interdiffusion of the solvents in the solvent mixture). The resulting two eigenvalues $\widehat{D}_{1}$ and $\widehat{D}_{2}$ are reported in Table 2 . These values in Table 2 are calculated by averaging the fitting results of the experiments performed at three temperature differences, 20,15 and $10 \mathrm{~K}$, and the uncertainties are the corresponding standard deviations. These values are comparable with those obtained in our previous study [14] for a similar polymeric mixture prepared with the same components of this study but a slightly different polymer molecular weight, and with those reported in $[43,45]$ for the diluted binary mixture of PS-toluene and in $[44,46]$ for the binary solvent mixture of toluene-n-hexane. Furthermore, the value of the $D r$ number can be determined as the ratio between the two eigenvalues, resulting in $\mathrm{Dr}=8.4$ (see Table 2). This value totally justifies the selection of the polymeric mixture as a suitable sample for experimentally studying well-separated concentration modes.

In a similar way, in order to get information about the thermal diffusivity $a_{T}$ of the ternary mixture, one can try to fit the data points of the thermal mode with the theoretical predictions 
for thermal NEFs only. In the diffusive regime, the decay time of the thermal mode can be expressed as [54]:

$$
\tau_{t h}(q)=1 /\left(a_{T} q^{2}\right)
$$

and the fitting of temperature NEFs for $q>90 / \mathrm{cm}$ in Fig. 3 provides the thermal diffusivity value reported in Table 2. This result is again comparable with the one obtained in previous studies for a similar polymeric mixture [14] as well as with that reported in [46] for the equimolar toluene-n-hexane binary solvent mixture.

From the two diffusion eigenvalues and the thermal diffusivity, the $L e_{i}$ numbers can be determined, which are also listed in Table 2.

\section{Table 2: Diffusion coefficient eigenvalues, $L e_{i}$ and Dr numbers of the polymeric ternary} mixture

\begin{tabular}{|c|c|}
\hline Parameter & Value \\
\hline$\widehat{D}_{1}$ & $(3.8 \pm 0.1) \times 10^{-6} \mathrm{~cm}^{2} / \mathrm{s}$ \\
\hline$\widehat{D}_{2}$ & $(3.1 \pm 0.1) \times 10^{-5} \mathrm{~cm}^{2} / \mathrm{s}$ \\
\hline$a_{T}$ & $(8.5 \pm 0.2) \times 10^{-4} \mathrm{~cm}^{2} / \mathrm{s}$ \\
\hline$L e_{1}$ & $227 \pm 12$ \\
\hline$L e_{2}$ & $27 \pm 2$ \\
\hline$D r$ & $8.4 \pm 0.6$ \\
\hline
\end{tabular}

\section{Discussion}

\section{Comparison between experimental and theoretical decay times}

As described in section 3.2, Martínez-Pancorbo et al. [40] presents the analysis of the fluctuations of two independent concentrations together with the effect of buoyancy, but considering neither the effect of confinement nor the presence of temperature and velocity 
fluctuations. Consequently, the coupling between modes which is known to be important at small wave numbers, as demonstrated for binary mixtures [43], is not taken into account. In order to be insensitive to confinement effects the experiments reported here have been performed in a thick cell with vertical dimension of $L=5 \mathrm{~mm}$. In these conditions, the effect of confinement should not be visible in the investigated wave number range. In fact, confinement effects appear only at dimensionless wave numbers $\tilde{q}=q L<5$, corresponding here to dimensional wave numbers $q<10 / \mathrm{cm}$, at the very bottom of the investigated range of $q[41,42]$.

Regarding coupling effects, it is mandatory to consider them whenever oscillations are visible in the experimental c-SF at small $q$ (see Fig. 2). The complete FHD theory, including the presence of velocity and temperature fluctuations and the various couplings among all modes, has been previously published by us [43] for the case of binary mixtures, as summarised in section 3.3. The results of [43] can be very useful for discussing the impact of the velocitytemperature coupling on the measured decay times.

In the following, we compare the experimental values of decay times at different wave numbers (obtained by fitting the c-SF through Eqs. 3 and 7 or 16), with the two published models [40, 43]. This allows to understand the impact of the different effects on the measured c-SF, and to evaluate if a more complete theoretical framework is needed or if on the contrary, the available theories can be simply applied in different wave number ranges in order to extract the transport properties of the investigated ternary mixture. As described in the previous section, at least the diffusion coefficients can be safely determined by fitting decay times at large wave numbers, where NEFs are dominated only by the diffusion phenomenon. The measurement of the Soret coefficient is more complicated as it requires the analysis of the effect of gravity [6], or other suitable analysis [55]. The simultaneous and independent characterization of the two Soret coefficients of a ternary mixture requires a complete knowledge of the diffusion matrix 
including the cross-diffusivities. This has not been achieved yet by Shadowgraphy and is the

528 ultimate goal of our current research. Although we estimate that this can be done with one-

529 wavelength Shadowgraphy, a more precise information can be obtained with a two-wavelength

530 version of the technique. The two-wavelength Shadowgraphy is currently being developed in

531 our lab, also in relation to the future space experiments of the NEUF-DIX project [28].

532 Figure 4 shows the experimental decay times and the oscillation frequency obtained from the

533 c-SF (data points are the same as in Fig. 3), together with theoretical predictions provided either by Eq. 8 (dashed yellow curves) or by numerically solving Eqs.14-15 (solid green curves).

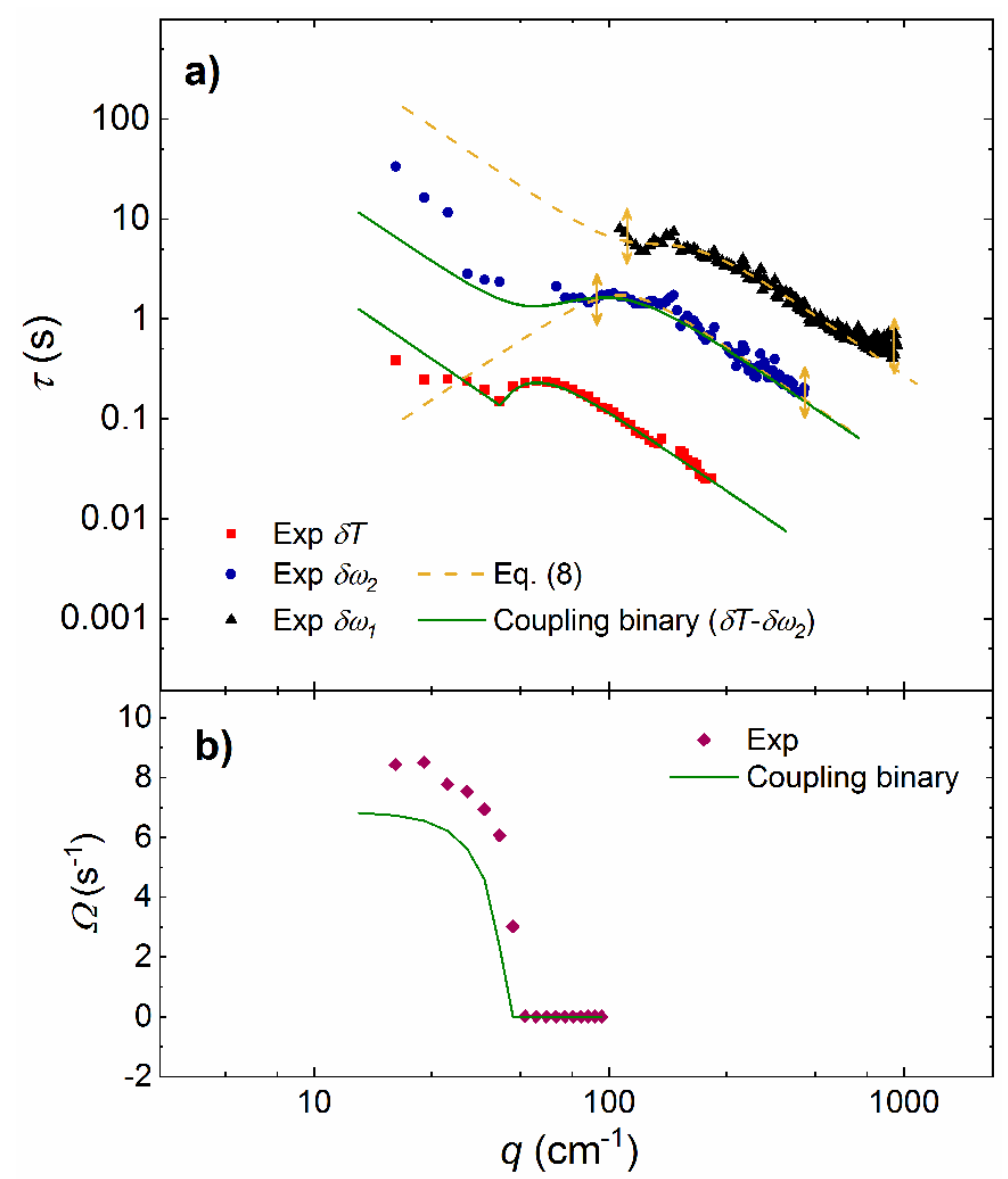

Figure 4: a) Decay times and b) oscillation frequency of NEFs on the ternary mixture of PS- 
represent the numerical calculations of Eqs. 8 (dashed lines) and the theory developed for binary mixtures including the effect of propagating modes (solid lines).

\section{The experimental data are fitted to Eq. 8 by fixing the diffusion matrix eigenvalues $\left(\widehat{D}_{1}\right.$ and $\left.\widehat{D}_{2}\right)$} to the corresponding values presented in Table 2 . The parameters obtained from this fitting to Eq. 8 are: $R a_{i} \widehat{D}_{i}=(260 \pm 40) \mathrm{cm}^{2} / \mathrm{s}$, and $R a_{S, t e r}=(4.6 \pm 1.0) \times 10^{7}$. As visible in Fig. $4 \mathrm{a}$, the experimental results suggest that the available theory for the ternary mixture reported by Martínez Pancorbo et al. [40] cannot describe the behaviour of c-NEFs in the present experiment for the whole range of wave numbers. The theory provides suitable predictions for intermediate and especially for large wave numbers, but fails in the limit of small $q$ (in particular for the fastest concentration mode) where the effect of the coupling with temperature fluctuations, and the consequent appearance of propagating modes in the experimental structure function become significant (see section 3.3) [43]. Hence, investigation of the coupling with temperature fluctuations is required for a full understanding of the present experimental results. We proceed into two steps: first we compare with the published theory [43] for the binary mixture case (one concentration mode) as reviewed in section 3.3; second we compare with the theory for ternary mixtures (two concentration modes) as briefly presented here in section 3.4. This allows appreciating the features that are genuinely related to the ternary mixture case.

As already mentioned, Fig. 4 shows, superposed on the experimental data, the theoretical predictions (green solid lines) of the theory [43] with one concentration mode. The theoretical curves have been obtained numerically (solving Eqs. 14 and 15) by keeping constant the Lewis number $L e=27$ to the same value of $L e_{2}$ shown in Table 2, and varying the values of $\operatorname{Pr}$ and $\psi$ to best fit the data. The solutal Rayleigh number $R a_{S, 2}=-\left(q_{S, 2}^{*} L\right)^{4}$ is also kept constant at $-5.8 \times 10^{6}$, after evaluating the value of the solutal rolloff wave number $q_{S, 2}^{*}$. This value represents the wave number where the fluctuation $\delta \omega_{2}$ behaviour changes from diffusive to 
gravitational [6]. The resulting values for the free fitting parameters are the following: $\operatorname{Pr}=$ 9.0 and $\psi=0.9$. As it can be observed in Fig. 4a, the decay times provided by the numerical solution agree very well with the experimental decay times of the temperature NEFs in the whole range of wave numbers. However, for the diffusion mass mode, at the smallest wave number range, the agreement is not as good as for the temperature, and a slowing-down of the experimental decay times is visible in comparison to the corresponding numerical solution (green solid line). The theoretical results of the oscillation frequency are likewise similar to the experimental data (see Fig. 4b). This last comparison between the experimental and theoretical 573 decay times shows the importance of including temperature fluctuations in the theoretical 574 analysis.

575 Next, we finally take into account the more complete theory of NEFs, which includes the coupling of thermal and viscous fluctuations to the two independent concentration modes present in a ternary mixture, as briefly described in section 3.4. The comparison between experimental and theoretical decay times is shown in Fig. 5. The effect of considering the two concentration modes and the associated couplings is evident by comparing Fig. 4 and 5. 


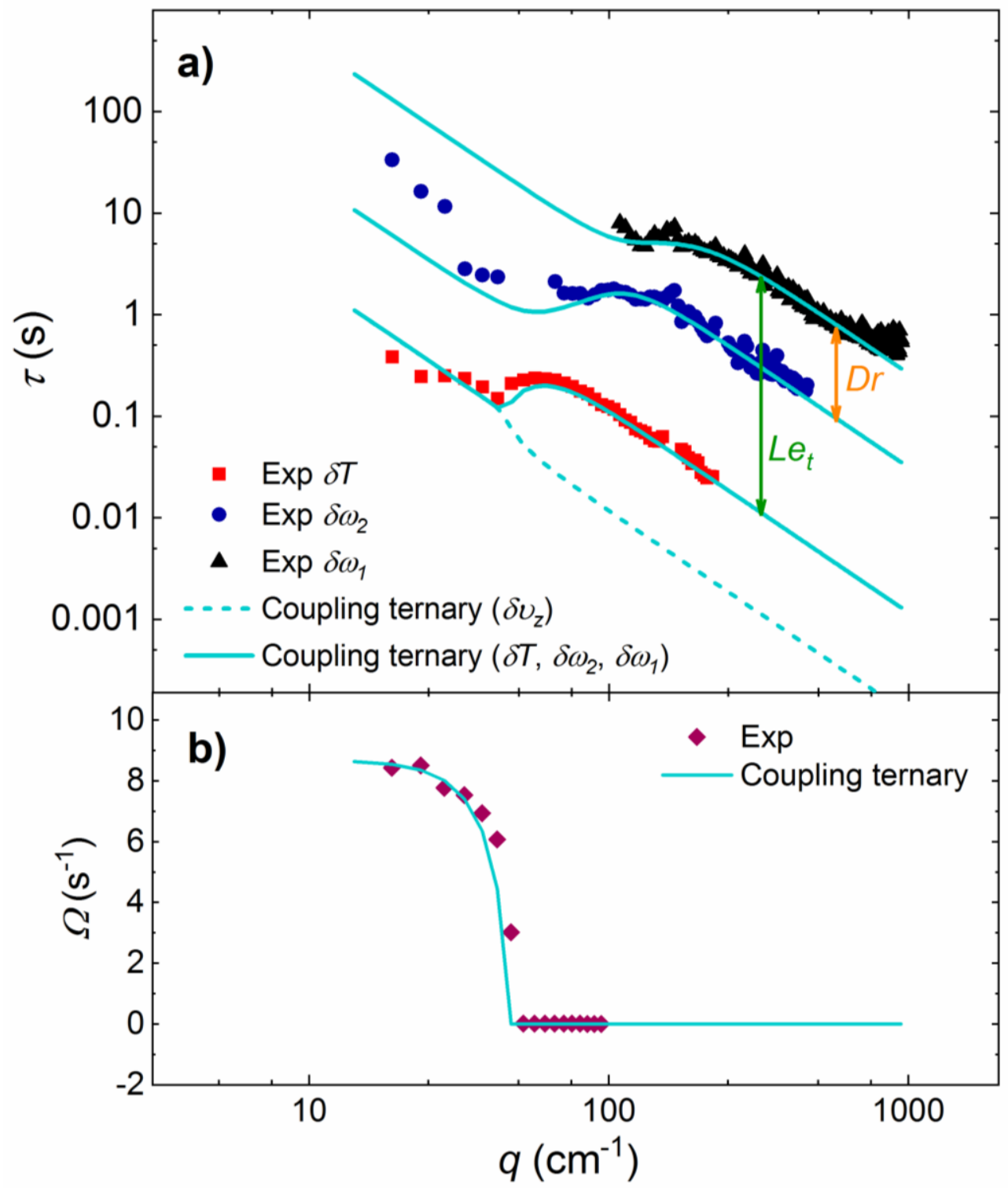

581

582

583

Figure 5: a) Decay times and b) oscillation frequency of NEFs on the ternary mixture of PStoluene-n-hexane in a thermodiffusion experiment performed at temperature difference of $20 \mathrm{~K}$, as a function of the wave number q. Experimental data are shown by points, while lines represent the theory developed for ternary mixtures including the effect of propagating modes.

The theoretical curves shown in Fig. 5 have been obtained numerically (solving Eqs. 18 and 19) by keeping constant $L e_{t}=227$ and $D r=8.4$ (the $L e_{1}$ and $D r$ values shown in Table 2), and varying the values of $\operatorname{Pr}, \psi_{1}^{\prime}$ and $\psi_{2}^{\prime}$ to best fit the data. Additionally, the term $\operatorname{Pr} L e_{t}{ }^{2} R a=$ 
$\alpha g L^{4} \nabla T / \widehat{D}_{1}^{2}$ appearing in the matrix $G_{t}(\tilde{q})$ of Eq. 18 is also kept fix at $1.6 \times 10^{11}$, considering

591 for this number the $\alpha$ value of Table 1, the $\widehat{D}_{1}$ value of Table 2, and the applied temperature 592 gradient. The resulting values for the free fitting parameters are the following: $\operatorname{Pr}=10, \psi_{1}^{\prime}=$

593

0.7 and $\psi_{2}^{\prime}=0.4$. The theoretical results show that for large wave numbers $\left(q \geq 47 \mathrm{~cm}^{-1}\right)$, four real solutions are obtained, demonstrating that all the modes are decoupled in this wave number range. The velocity mode cannot be observed experimentally, as it does not give rise to refractive index fluctuations. For small wave numbers $\left(q<47 \mathrm{~cm}^{-1}\right)$, two real solutions and a pair of complex conjugate ones are obtained numerically, indicating that velocity and temperature fluctuations are mixed at small $q$. This mixing induces the appearance of propagating modes, observed in the experimental c-SF of Fig. 2 as oscillations at small $q$.

Applying the full theory of section 3.4, we found a reasonable agreement between the experimental and the theoretical data for the three modes, especially for large and intermediate wave numbers. However, we would like to point out that the numerical values of $\operatorname{Pr}, \psi_{1}^{\prime}$ and $\psi_{2}^{\prime}$ must be taken into account with caution as the fitting procedure is not robust. The focus here is mainly on the qualitative understanding of the behaviour of the different modes in a ternary mixture when the coupling among them is included.

One relevant point is the slowing-down of the two concentration modes and their consequent diffusive-like behaviours at very small wave numbers (see Fig. 5a). In addition, it is worth noting the good agreement obtained for the frequency of the propagating mode, as can be seen in Fig. 5b. It clearly improves the results of the binary model shown in Fig. 4b.

Of course, the effect of confinement should be also included in the theory if one wants to describe layers of ternary mixtures of any vertical thickness. Unfortunately, this additional development requires more effort and no analytical solution can be obtained.

\section{Conclusions}


The concentration and temperature NEFs in a thermodiffusion experiment of a ternary mixture composed of polystyrene, toluene and n-hexane are characterized by dynamic shadowgraphy. The decay times of the NEFs are determined and three modes are clearly distinguished. The fastest decay times are associated to the temperature NEFs, the intermediate ones to the concentration NEFs of the molecular solvent and the slowest ones to the concentration NEFs of the polymer. To encompass the very different decay times (i.e. large range of correlation times), images are acquired at various frequencies, and the corresponding SFs are merged into the so-called c-SF by following the described concatenation procedure.

In order to exploit the obtained decay times, first, they are fitted with a simple diffusive model at large wave numbers to obtain the diffusion eigenvalues $\widehat{D}_{1}$ and $\widehat{D}_{2}$ and the thermal diffusivity $a_{T}$. Secondly, the theory developed for the two concentration modes of a ternary mixture in the presence of gravity is compared with the experimental results for the first time. The experimental decay times of the concentration modes at intermediate and large wave numbers appear to be in good agreement with this theoretical model. However, for smaller wave numbers, a slowing-down of the experimental decay times of NEFs is detected with respect to the predictions of that theory. Due to the large thickness of the thermodiffusion cell, confinement effects cannot be considered as the origin of such discrepancy. In addition, evidence of propagating modes on the structure function suggests that the aforementioned slowing-down could be originated by the coupling of the temperature and viscous modes to the concentration ones. Comparison with the decay times from the theory developed to describe such coupling in a binary mixture shows a better agreement for the thermal and the faster concentration modes at intermediate wave numbers. This positive outcome lead us to further develop the theory of NEFs in the case of ternary mixtures, by including the coupling of velocity, temperature and the two concentration modes under strong gravitational stabilization 
650

651

652

653

654

655

656

657

658

659

660

661

662

663

and for large size fluctuations (small wave numbers). A better qualitative agreement is found between the new theoretical development and the experimental data points.

\section{Acknowledgements}

The authors of the University of Pau kindly thank the financial support from the Centre National d'Etudes Spatiales (CNES) and from the funding partners of the Industrial Chair CO2ES: E2SUPPA, TOTAL, CNES and BRGM. L. García-Fernández gratefully acknowledges the CNES for the post-doctoral research grant. The research at the Complutense University was supported by grant ESP2017-83544-C3-2-P of the Spanish Agencia Estatal de Investigación.

\section{Authors contribution statement}

The experimental measurements and data analysis were performed by L. García-Fernández, H. Bataller and F. Croccolo. P. Fruton contributed to the concatenation procedure of the Structure Function. J.M. Ortiz de Zárate and L. García-Fernández focused on the new theoretical development presented in section 3.4. All the authors contributed to the discussion of the results and in writing the manuscript.

\section{References}

[1] F. Montel, J. Bickert, A. Lagisquet and G. Galliéro, J. Petrol. Sci. Eng., 58, 391 (2007).

[2] H. Guo, Q. Zhou, Z. Wang and Y. Huang, Int. J. Heat Mass Tran., 117, 966 (2018).

[3] J. M. Ortiz de Zarate and J. V. Sengers, Hydrodynamic Fluctuations in Fluids and Fluid Mixtures, Elsevier, Amsterdam, (2006).

[4] F. Croccolo, J. M. Ortiz de Zarate and J. V. Sengers, Eur. Phys. J. E, 39, 125 (2016).

[5] A. Vailati and M. Giglio, Nature, 390, 262 (1997). 
664

665

666

667

668

669

670

671

672

673

674

675

676

677

678

679

680

681

682

683

684

685

686

687

[6] F. Croccolo, H. Bataller and F. Scheffold, J. Chem. Phys., 137, 234202 (2012).

[7] C. Giraudet, H. Bataller and F. Croccolo, Eur. Phys. J. E, 37, 107 (2014).

[8] C. Ludwig, Sitzungsbur. Osterr. Akad. Wiss., Math.-Nat. Kl., 20, 539 (1856).

[9] C. Soret, Arch. Sci. Phys. Nat. Geneve, 2, 48 (1879).

[10] W. Köhler and K. I. Morozov, J. Non-Equil. Thermody., 41, 151 (2016).

[11] F. Croccolo and D. Brogioli, Appl. Optics, 50, 3419 (2011).

[12] F. Croccolo, D. Brogioli, A. Vailati, M. Giglio and D. S. Cannell, Phys. Rev. E, 76, 041112 (2007).

[13] S. P. Trainoff and D. S. Cannell, Phys. Fluids, 14, 1340 (2002).

[14] H. Bataller, T. Triller, B. Pur, W. Köhler, J. M. Ortiz de Zarate and F. Croccolo, Eur.

Phys. J. E, 40, 35 (2017).

[15] I. Lizarraga, C. Giraudet, F. Croccolo, M. M. Bou-Ali and H. Bataller, Microgravity Sci. Tec., 28, 545 (2016).

[16] J. K. Platten, M. M. Bou-Ali, P. Costesèque, J. F. Dutrieux, W. Köhler, C. Leppla, S.

Wiegand and G. Wittko, Philos. Mag., 83, 1965 (2003).

[17] H. Bataller, C. Giraudet, F. Croccolo and J. M. Ortiz de Zárate, Microgravity Sci. Tec., 28, 611 (2016).

[18] M. M. Bou-Ali, A. Ahadi, D. Alonso de Mezquia, Q. Galand, M. Gebhardt, O. Khlybov, W. Köhler, M. Larranaga, J. C. Legros, T. Lyubimova, A. Mialdun, I. Ryzhkov, M. Z. Saghir, V. Shevtsova and S. Van Vaerenbergh, Eur. Phys. J. E, 38, 30 (2015).

[19] A. Ahadi, S. V. Varenbergh and M. Z. Saghir, J. Chem. Phys., 138, 204201 (2013).

[20] A. Mialdun and V. Shevtsova, J. Chem. Phys., 143, 224902 (2015).

[21] A. Mialdun, I. Ryzhkov, O. Khlybov, T. Lyubimova and V. Shevtsova, J. Chem. Phys., 148, 044506 (2018). 
688

689

690

691

692

693

694

695

696

697

698

699

700

701

702

703

704

705

706

707

708

709

710

711

712

[22] T. Triller, H. Bataller, M. M. Bou-Ali, M. Braibanti, F. Croccolo, J. M. Ezquerro, Q.

Galand, J. Gavaldà, E. Lapeira, A. Laverón-Simavilla, T. Lyubimova, A. Mialdun, J. M. Ortiz de Zárate, J. Rodríguez, X. Ruiz, I. I. Ryzhkov, V. Shevtsova, S. V. Vaerenbergh and W.

Köhler, Microgravity Sci. Tec., 30, 295 (2018).

[23] A. Mialdun, H. Bataller, M. M. Bou-Ali, M. Braibanti, F. Croccolo, A. Errarte, J. M.

Ezquerro, Y. Gaponenko, L. García-Fernández, J. J. Fernández, J. Rodríguez and V.

Shevtsova, Eur. Phys. J. E, 42, 87 (2019).

[24] G. Galliero, H. Bataller, J. P. Bazile, J. Diaz, F. Croccolo, H. Hoang, R. Vermorel, P. A. Artola, B. Rousseau, V. Vesovic, M. M. Bou-Ali, J. M. Ortiz de Zarate, S. Xu, K. Zhang, F. Montel, A. Verga and O. Minster, NPJ Microgravity, 3, 20 (2017).

[25] J. C. Legros, V. Vaerenbergh, D. S., Y. and F. Montel, Entropy, 198/199, 1 (1994).

[26] S. VanVaerenbergh, S. Srinivasan and M. Z. Saghir, J. Chem. Phys., 131, 114505 (2009).

[27] G. Galliero, H. Bataller, F. Croccolo, R. Vermorel, P.-A. Artola, B. Rousseau, V.

Vesovic, M. Bou-Ali, J. M. Ortiz de Zárate, S. Xu, K. Zhang and F. Montel, Microgravity Sci.

Tec., 28, 79 (2015).

[28] P. Baaske, H. Bataller, M. Braibanti, M. Carpineti, R. Cerbino, F. Croccolo, A. Donev,

W. Köhler, J. M. Ortiz de Zarate and A. Vailati, Eur. Phys. J. E, 39, 119 (2016).

[29] M. Braibanti, P.-A. Artola, P. Baaske, H. Bataller, J.-P. Bazile, M. M. Bou-Ali, D. S.

Cannell, M. Carpineti, R. Cerbino, F. Croccolo, J. Diaz, A. Donev, A. Errarte, J. M. Ezquerro,

Q. Galand, G. Galliero, Y. Gaponenko, L. García-Fernández, J. Gavaldá, F. Giavazzi, M.

Giglio, H. Hoang, W. Köhler, E. Lapeira, A. Laverón-Simavilla, I. Lizarraga, T. Lyubimova, S. Mazzoni, A. Mialdun, O. Minster, F. Montel, J. M. O. d. Zárate, J. Rodríguez, B.

Rousseau, X. Ruiz, I. I. Ryzhkov, M. Schraml, V. Shevtsova, C. J. Takacs, T. Triller, S.

VanVaerenbergh, A. Vailati, A. Verga, R. Vermorel, V. Vesovic, V. Yasnou, S. Xu, D. Zapf and K. Zhang, Eur. Phys. J. E, 42, 86 (2019). 
[30] P. Blanco, M. M. Bou-Ali, J. K. Platten, D. A. de Mezquia, J. A. Madariaga and C.

Santamaria, J. Chem. Phys., 132, 114506 (2010).

[31] A. Leahy-Dios, M. M. Bou-Ali, J. K. Platten and A. Firoozabadi, J. Chem. Phys., 122, $234502(2005)$.

[32] A. Königer, H. Wunderlich and W. Köhler, J. Chem. Phys., 132, 174506 (2010).

[33] A. Ahadi and M. Ziad Saghir, Eur. Phys. J. E, 38, 25 (2015).

[34] A. Mialdun, J. C. Legros, V. Yasnou, V. Sechenyh and V. Shevtsova, Eur. Phys. J. E, 38, $27(2015)$.

[35] M. Gebhardt and W. Kohler, Eur. Phys. J. E, 38, 24 (2015).

[36] Q. Galand and S. Van Vaerenbergh, Eur. Phys. J. E, 38, 26 (2015).

[37] O. A. Khlybov, Ryzhkov, II and T. P. Lyubimova, Eur. Phys. J. E, 38, 29 (2015).

[38] M. Larranaga, M. M. Bou-Ali, D. A. de Mezquia, D. A. Rees, J. A. Madariaga, C.

Santamaria and J. K. Platten, Eur. Phys. J. E, 38, 28 (2015).

[39] J. M. Ortiz de Zarate, C. Giraudet, H. Bataller and F. Croccolo, Eur. Phys. J. E, 37, 77 (2014).

[40] P. Martínez Pancorbo, J. M. Ortiz de Zarate, H. Bataller and F. Croccolo, Eur. Phys. J. E, 40, 22 (2017).

[41] C. Giraudet, H. Bataller, Y. Sun, A. Donev, J. M. Ortiz de Zárate and F. Croccolo, EPLEurophys. Lett., 111, 60013 (2015).

[42] C. Giraudet, H. Bataller, Y. Sun, A. Donev, J. M. Ortiz de Zarate and F. Croccolo, Eur. Phys. J. E, 39, 120 (2016).

[43] F. Croccolo, L. García-Fernández, H. Bataller, A. Vailati and J. M. Ortiz de Zarate, Phys. Rev. E, 99, 012602 (2019).

[44] D. Alonso de Mezquia, Z. Wang, E. Lapeira, M. Klein, S. Wiegand and M. Mounir BouAli, Eur. Phys. J. E, 37, 106 (2014). 
[45] J. Rauch and W. Köhler, J. Chem. Phys., 119, 11977 (2003).

739

[46] K. J. Zhang, M. E. Briggs, R. W. Gammon and J. V. Sengers, J. Chem. Phys., 104, 6881

740 (1996).

741 [47] D. Brogioli, A. Vailati and M. Giglio, Phys. Rev. E, 61, 1 (2000).

742 [48] F. Croccolo, D. Brogioli, A. Vailati, M. Giglio and D. S. Cannell, Appl. Optics, 45, 2166 743 (2006).

744 [49] G. Cerchiari, F. Croccolo, F. Cardinaux and F. Scheffold, Rev. Sci. Instrum., 83, 106101 745 (2012).

746 [50] L. Cipelletti and D. A. Weitz, Rev. Sci. Instrum., 70, 3214 (1999).

747 [51] J. M. Ortiz de Zarate, Eur. Phys. J. E, 42, 43 (2019).

748 [52] J. M. Ortiz de Zárate, J. L. Hita and J. V. Sengers, CR. Mecanique, 341, 399 (2013).

749 [53] A. Bardow, Fluid Phase Equilibria, 251, 121 (2007).

750 [54] P. N. Segrè, R. Schmitz and J. V. Sengers, Physica A, 195, 31 (1993).

751 [55] F. Croccolo, C. Giraudet, H. Bataller, R. Cerbino and A. Vailati, Microgravity Sci. Tec., $752 \quad 28,467(2016)$.

753

754

755

756 